\title{
Classification Theorem for Principal Fibre Bundles, Berry's Phase, and Exact Cycle Evolution
}

$$
\text { by }
$$

Arno Bohm, Louis J. Boya,

Ali Mostafazadeh, and Gerd Rudolph

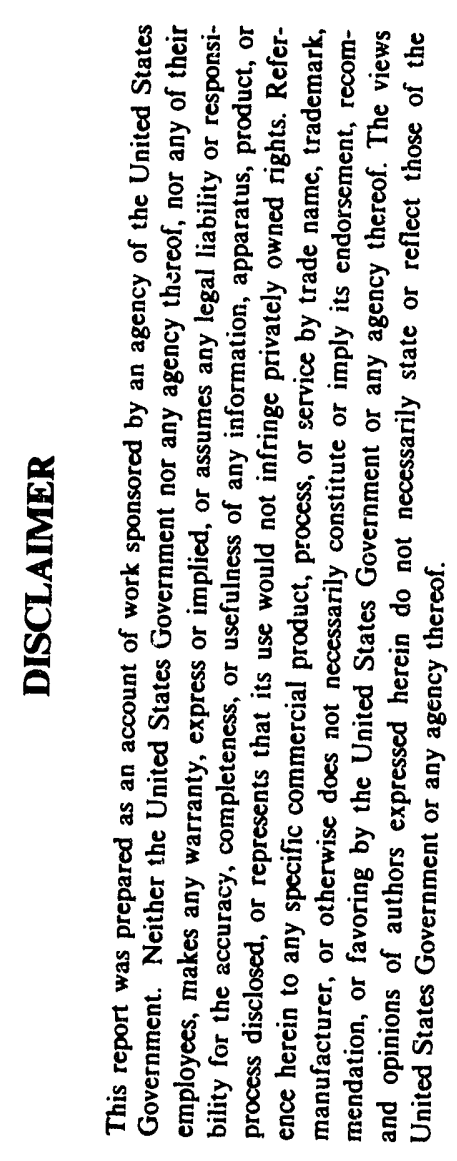

Center for Particle Physics University of Texas at Austin Austin, Texas $\mathbf{7 8 7 1 2}$ 


\title{
CLASSIFICATION THEOREM FOR PRINCIPAL FIBRE BUNDLES, BERRY'S PHASE, AND EXACT CYCLIC EVOLUTION
}

\author{
Arno BOHM, Louis J. BOYA *, Ali MOSTAFAZADEH, and Gerd RUDOLPH ${ }^{\dagger}$ \\ The University of Texas at Austin, Austin, Texas 78712
}

\begin{abstract}
We discuss the relation between the two mathematical interpretations of the geometric (Berry) phase, using either the fibre bundle over parameter space or over projective Hilbert space. It turns out that these two geometric constuctions are linked by the classification theorem for vector bundles. The classification theorem provides the means to classify the parameter space bundles for adiabatic evolution and for non-adiabatic cyclic evolution of the statevectors.
\end{abstract}

* Permanent address: Departamento de Fisica Teórica, Facultad de Ciencias, Universidad de Zaragoza. 50009 Zaragoza, SPAIN.

$\dagger$ Fachbereich Physik, Universitat Leipzig, 7010 Leipzig, Augustusplatz 10, GERMANY. 


\section{INTRODUCTION}

In the original geometric interpretation of the Berry phase by B. SIMON [1], a quantum system traversed a closed cycle in some parameter space $M: t \rightarrow x(t), 0 \leq t \leq T$, $x(0)=x(T)$. To each point $x \in M$ is associated an instantaneous Hamiltonian $H(x)$ and a one-dimensional eigenspace of $H(x)$. In this way one gets a line bundle $L:\left[x, L_{x}\right]$, where $L_{x}=\{c|n, x>| c \in \mathbb{C}\}$ is the one-dimensional eigenspace of a non-degenerate eigenvalue $E_{n}(x)$ of $H(x), H(x)|n, x\rangle=E(x) \mid n, x>$. If $\mid \psi(t)>$ is the state vector that develops according to the time dependent Hamiltonian $H(x(t))$ adiabarically, i.e.

$$
|\psi(t)><\psi(t)|=|n, x(t)><n, x(t)|
$$

for all $t$ from an eigenstate $|\psi(0)\rangle=|n, x(0)\rangle$, then $\mid \psi(T)>$ picks up, in addition to the usual "dynamical" phase angle $\delta=-\frac{1}{\hbar} \int^{T} E_{n}(t) d t$ a "geometrical" phase angle $\gamma$ :

$$
|\psi(T)\rangle=e^{i(\delta+\gamma)}|\psi(0)\rangle
$$

Since the level crossing loci are to be excluded, $M$ is in general non-contractible. This allows for $L$ to be a non-trivial line bundle and consequently any connection on $L$ develops a $U(1)$ holonomy, which Simon naturally identified with the phase $e^{i \gamma}$ previously found by BERRY [2].

A few years later, AHARONOV and ANANDAN (A-A) [3] suggested to consider the loop in the state space, namely the projective Hilbert space

$$
P(\mathcal{H})=\{|\psi\rangle\langle\psi|:| \psi\rangle \in \mathcal{H}\}=\mathbb{C} P(\infty)
$$

Cyclic evolutions are then given by those solutions $\psi$ of the Schrödinger equation with time dependent Hamiltonian $H(t)$, for which the state $|\psi(t)><\psi(t)|$ traverses a closed loop.

$$
\mathcal{C}: t \rightarrow|\psi(t)><\psi(t)| \quad, \quad 0 \leq t \leq T \quad \text { with } \quad|\psi(T)><\psi(T)|=|\psi(0)><\psi(0)|
$$

The cyclic adiabatic evolution is then a limiting case of an evolution given by a closed loop in state space. Now the natural principal bundle of norm-one state vectors is the universal 
classifying bundle $\eta: U(1) \rightarrow S^{\infty} \rightarrow P(\mathcal{H})$ which is non-trivial and possesses a natural connection-cum-curvature. A-A showed that the holonomy of the (metric) connection identifies with Berry's [2] in the adiabatic limit. The naturality and the uniqueness of this geometric construction has been shown by several people [4], [5] including some of us $[6]-[9]$.

This paper is devoted to explain in detail the relation between the two approaches. In section II and III, we discuss the conventional complex Hilbert cpace, and the real Hilbert space cases respectively. Here the eigenvalue $E_{n}(x)$ is assumed to be non-degenerate. The generalization to degenerate cace and the quaternionic case of $S p(1)$ bundles are considered in section IV. This section also summarizes the geometric aspects of the problem. Essentially, the pull-back of the universal connection on $\eta$ to the bundle $L$ gives the Berry phase for adiabatic evolutions. In section $\mathrm{V}$, the ideas developed in the previous sections are used to treat the more realistic case of non-adiabatic evolution of statevectors. For a particular class of quantum systems, our approach leads to the construction of non-adiabatic (Simon's) bundle over the parameter space. The connection on this bundle yields the geometric (non-adiabatic Berry) phase.

The topological classification of the problem of geometric phase was also discussed by KIRITSIS [10]. However, we offer an alternative mathematical treatment which is more direct in method and suggested by the physics of the problem. ${ }^{1}$

\section{UNIVERSAL BUNDLES: THE COMPLEX CASE, NON-DEGENERATE EIGENVALUES}

Let $M$ be an oriented, smooth, compact manifold, serving as the parameter space for the hermitian operator $H(x)$, i.e. $H(x)$ depends continuously on $x \in M$. Let $E_{n}(x)$ be an isolated non-degenerate eigenvalue of $H(x)$. Then the association $x \rightarrow|n, x\rangle$ given by $H(x)\left|n, x>=E_{n}(x)\right| n, x>$ defines a line bundle $L$, over $M$ with fibers:

$$
L_{x}=\left\{\left|\psi_{x}\right\rangle:\left|\psi_{x}\right\rangle=c|n, x\rangle, c \in \mathbb{C}\right\} \cong \mathbb{C}
$$

Here $|n, x\rangle$ denotes a normalized eigenvector of $H(x)$ which is a single valued function of

1 There has been questions regarding the validity of some of the results indicated in [10], these have been pointed out by Douglas and Rutherford [11]. 
$x$. Let $C:[0, T] \rightarrow M$ be a continuous, closed loop, i.e. $C(0)=C(T)=x_{0}$, and consider the normalized solution $\mid \psi[C(t)]>$ of the Schrödinger equation

$$
i \hbar \frac{d}{d t}|\psi[C(t)]\rangle=H(C(t))|\psi[C(t)]\rangle
$$

Not all solutions of (4) are cyclic solutions, i.e. fulfill (2). Those $\mid \psi[C(t)]>$ which fulfill (2) are called dynamical lift of (2).

In the adiabatic limit, the situation contemplated by BERRY [2] and SIMON [1], [12], where an initial eigenstate of $H(C(0))$ remains always an eigenstate of $H[C(t)]$ i.e. where (1) is fulfilled, the evolution is always cyclic in the sense of (2). The statevector $\mid \psi[C(t)]>$ does not traverse a closed loop but picks up a phase:

$$
\left|\psi[C(T)]>=e^{i(\delta+\gamma)}\right| \psi[C(0)]>
$$

where $\delta=-\frac{1}{\hbar} \int E[C(t)] d t$ is the dynamical phase angle and $\gamma=\gamma(C)=\int_{S} \Omega$ is the geometric (Berry) phase angle, here, $\Omega=d A, \partial S=C$ and $A=i\langle n, x|d| n, x\rangle$ is the Berry connection one form.

AHARONOV and ANANDAN [3], [4] and ANANDAN and STODOLSKY [13] realized that instead of considering a closed loop in parameter space, $\mathrm{C}$, one could consider the closed loop in state space, $\mathcal{C}: t \rightarrow \mathcal{C}(t)=|\psi[C(t)]><\psi[C(t)]| \in P(\mathcal{H})$ and drop the adiabaticity condition. They showed that a geometric phase is also associated with a cyclic path in $P(\mathcal{H})$.

A relevant task is then to investigate the relation between the Berry-Simon (B-S) picture and Aharonov-Anandan's picture.

Let $\eta$ be the $U(1)$ principal fibre bundle (PFB) of A-A

$$
\eta: U(1) \rightarrow S^{2 N+1} \rightarrow \mathbb{C} P(N) \quad, \quad N \rightarrow \infty
$$

and $E$ its associated line bundle. Here

$$
\eta_{|\psi><\psi|}=\left\{e^{i \delta}|\psi>| e^{i \delta} \in U(1)\right\} \simeq U(1)
$$

is the fibre over $|\psi><\psi| \in P(\mathcal{H})=\mathbb{C} P(\infty)$. The fibres of $E$ are:

$$
E_{|\psi><\psi|}=\{c|\psi>| c \in \mathbb{C}\}
$$


Also define $\lambda$ to be the associated $U(1)$ PFB of the line bundle $L$ of B-S.

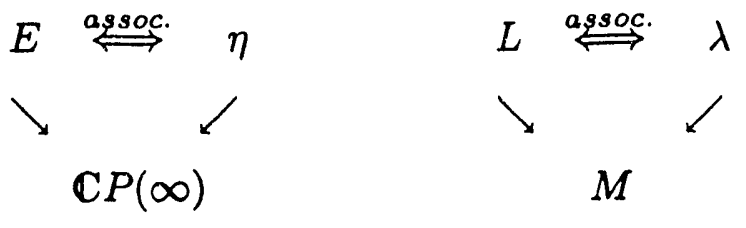

The relation between $\eta$ and $\lambda$ (alternatively $E$ and $L$ ) is the following: $\eta(E)$ is the universal classifying bundle for $U(1)$ PFB's ( $\mathbb{C}$-line bundles); see e.g. [14]§5; [15]§19; or [16]. The classifying theorem for $U(1)$ PFB's states that any $U(1)$ PFB $\xi$ over $M$ is equivalent (isomorphic) [15] to the pull-back bundle $f^{*}(\eta)$ for some continuous function $f: M \rightarrow$ $\mathbb{C} P(\infty)$, i.e. the following diagram is commutative:

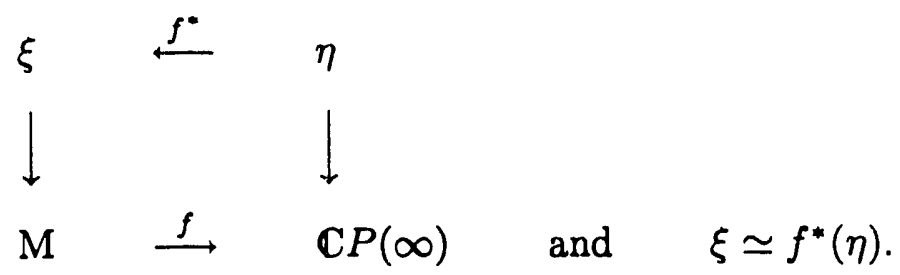

Furthermore, $\xi \simeq f^{*}(\eta)$ depends only on the homotopy class $[f] \in[M, \mathbb{C} P(\infty)]$ of $f$, i.e. homotopic maps induce equivalent (isomorphic) bundles. Now, taking $\xi=\lambda$ one has the desired relation. Similarly, for the associated line bundles the following commutative diagram summarizes the classification theorem for line bundles:

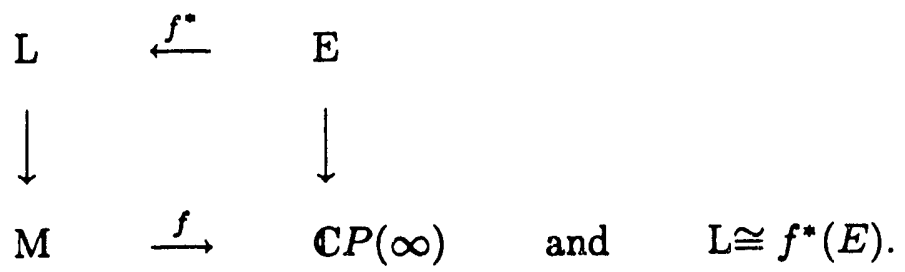

Since the induced bundle $f^{*}(\eta)\left(f^{*}(E)\right)$ depends on the equivalence class $[f] \in[M, \mathbb{C} P(\infty)]$, it is the topology of $M$ which determines all possible $U(1)$-bundles ( $\mathbb{C}$-line bundles) over $M$. For instance, if $f: M \rightarrow \mathbb{C} P(\infty)$ is homotopic to a constant map, then one will obtain the trivial bundle; $\lambda \simeq f^{*}(\eta) \simeq M \times U(1)\left(L \cong f^{*}(E) \cong M \times \mathbb{C}\right) .^{2}$

2 Note that the triviality of a bundle does not necessarily imply that the holonomy group is trivial. This is the very reason why the Berry phase is called the "geometric phase", and not the "topological phase". 
The topology of $\lambda(L)$ then depends on $[f] \in[M, \mathbb{C} P(\infty)]$. The existence of a one-toone correspondence between $[M, \mathbb{C} P(\infty)]$ and $H^{2}(M, \boldsymbol{z})$, implies a one-to-one correspondence between the set of (equivalence classes of) $U(1)$ PFB's, or equivalently the set of (isomorphism classes of) $\mathbb{C}$-line bundles, over $M$ and $H^{2}(M, \mathbb{Z})$. In fact the image of a bundle in $H^{2}(M, \mathbb{Z})$ is the first Chern class [18]. ${ }^{3}$ The fact that it is the second cohomology group of $M$ which matters, is a direct consequence of $\mathbb{C} P(\infty)$ being an Eilenberg-McLane space, namely $K(\mathbb{Z}, 2)$, i.e. $\pi_{2}(\mathbb{C} P(\infty))=\mathbb{Z}$ and $\pi_{n}(\mathbb{C} P(\infty))=\{0\}$ for $n \neq 2[19],[15]$. This was actually the starting point of our original analysis.

A simple physically non-trivial example is the following [2] [20]: Let the Hamiltonian be a $2 \times 2$ hermitian matrix:

$$
H(x)=\left(\begin{array}{cc}
a & b-i c \\
b+i c & d
\end{array}\right) \quad a, b, c, b \in \mathbb{R}
$$

The parameter space of simple eigenvalues if $M=\mathbb{R}^{4}-\ell$, where $\ell$ is the line $b=c=0, a=d$. The excluded line "digs out" a cylinder in $\mathbb{R}^{4}$ with section $S^{2}$, hence $M$ has the homotopy type of $S^{2}$. Therefore, one has

$$
\mathbb{Z} \cong H^{2}\left(S^{2}, \mathbb{Z}\right) \cong\left[S^{2}, \mathbb{C} P(\infty)\right] \equiv \pi_{2}(\mathbb{C} P(\infty)) \cong \mathbb{Z}
$$

which allows for non-trivial bundles over $M$. The "unit" bundle in this $\mathbb{Z}$-family is the lamous Hopf (beta) bundle: 4

$$
\hat{\beta}: U(1) \rightarrow S^{3} \rightarrow \mathbb{C P}(1)
$$

In general, the following question arises: "For a given Hamiltonian $H(x), x \in M$, what is the map $f: M \rightarrow \mathbb{C} P(\infty)$ that determines the bundle $\lambda \simeq f^{*}(\eta)$ ?" The answer is that $f$ is fixed by $H$, namely, $f(x)=|n, x><n, x|=\left|\psi_{x}><\psi_{x}\right| \in \mathbb{C} P(\infty)$ where $\left\|\mid \psi_{x}>\right\|=1$ and it satisfies $H(x)\left|\psi_{x}>=E(x)\right| \psi_{x}>$.

3 One can directly relate the isomorphism classes of $\mathbb{C}$-line bundles to $\breve{H}^{2}(M, \mathbb{Z})$ as described by Kostant [17] where " " means Čech cohomology. However, it is a well-known fact that $\breve{H}^{q}(M, \mathbb{Z}) \cong H^{q}(M, \mathbb{Z})$ for all $q[18]$.

4 The Hopf (beta) bundle appears in many physical problems e.g. in the VORTEX [21] and the MAGNETIC MONOPOLE [22]. The latter is specially noteworthy, and the continuous appearance of the magnetic monopole field in the context of Berry phase is precisely for this reason. 
The relation among the bundles also carries over to their connections, namely there is a "universal connection" on $\eta,[23]$, which pulls-back to $\lambda$. The pull-back is induced by the map $f: M \rightarrow \mathbb{C} P(\infty)$. We will give a more detailed discussion of the relation between the connections of these bundles in section IV. In particular we will show that the connection 1-form of the universal A-A bundle $\eta$ "corresponds" to the connection 1-form of the B-S parameter space bundle.

\section{THE REAL CASE, NON-DEGENERATE EIGENVALUES}

If all pertinent operators commute with the time reversal operator $T$, and if in addition $T^{2}=+1$, then the Hilbert space can be taken over the field of real numbers. The associated projective space $P(\mathcal{H})$ is $\mathbb{R} P(\infty)$, and the corresponding bundles have $O(1)=\mathbb{Z}_{2}$ as their structure group. Thus the geometric phase angle is in general a multiple of $\pi$. Actually this was first discovered by HERZBERG and LONGUET-HIGGINS [24], in their study of polyatomic molecules long before Berry's work. ${ }^{5}$

The simplest parameter space is obtained by the simple symmetric real $2 \times 2$ matrices $\left(\begin{array}{l}a b \\ b c\end{array}\right)$ with $a \neq c$ or $b \neq 0$. Here, $M=\mathbb{R}^{3}-\ell^{\prime}$ where $\ell^{\prime}$ is the line $b=0$ and $a=c$ in $\mathbb{R}^{3}[21]$. It has the homotopy type of $S^{1}$.

Now the corresponding A-A bundle $\eta$ is the universal classifying bundle for $O(1)$ PFB's. The state space $\mathbb{R} P(\infty)$ is now the Eilenberg-McLane space $K\left(\mathbb{Z}_{2}, 1\right)$ and all real PFB's ( $\mathbb{R}$-line bundles) are classified by the first Stiefel-Whitney class $w_{1} \in H^{1}\left(M, \mathbb{Z}_{2}\right)$ [15]. Therefore, we expect non-trivial $\mathbb{Z}_{2}$-bundles over non-simply connected parameter manifolds. For example, for $M=S^{1}$ introduced above, $\pi_{1}(M)=\pi_{1}\left(S^{1}\right)=\mathbb{Z}$, so one may have nontrivial bundles.

We would like to remark that in this case the holonomy does not come from a connection. Since the parallel translation is unique on bundles with discrete fibres, the connection is flat and a flat connection produces trivial restricted holonomy [25, ch. II]. Thus one gets a "phase" only for non-contractible loops. The situation is similar to parallel transporting along a sectional loop on a cone: although the surface is developable, the holonomy is nonzero, and given by the cone angle.

5 The resulting $e^{i n \pi}= \pm 1$ sign is sometimes referred to as "Longuet-Higgins" charge. 


\section{The DEGENERATE CASE}

We shall now discuss the case that the Hamiltonian $H(x)$, which depends upon a finite number of parameters $x=\left(x^{1} \ldots x^{m}\right) \in \tilde{M}$ has degenerate eigenvalues. Let $E_{n}(x)$ denote the $n$-th eigenvalue of $H(x)$ which is $\mathcal{N}$-fold degenerate and let us assume that level crossing occurs on a subset $\mathrm{N}$ of the parameter space $\tilde{M}$. The restricted parameter space $M \equiv \tilde{M} \backslash N$ has then (in general) nontrivial homotopy type. The observables like $H(x)$ and their eigenvalues like $E_{n}(x)$ must be (per assumption) single valued functions of the parameter $x \in M$. The $n$-th energy eigenstate, which is described by the $\mathcal{N}$-dimensional projection operator $\Lambda_{n}(x) \equiv \Lambda(x)$ is a single valued function of the continuously varying parameters $x \in M$. The basis system of orthonormal eigenvectors $\left\{\mid \chi^{i}(x)>: i=1,2, \ldots \mathcal{N}\right\}$ of $H(x)$ need not be single valued to span the energy eigenspace $\mathcal{H}_{n}(x)=\Lambda_{n}(x) \mathcal{H}$

$$
\begin{aligned}
H(x) \mid \chi^{i}(x)> & =E_{n} \mid \chi^{i}(x)>\quad, \quad i=1 \ldots \mathcal{N} \\
\Lambda_{n}(x) & =\sum_{i=1}^{\mathcal{N}}\left|\chi^{i}(x)><\chi^{i}(x)\right|
\end{aligned}
$$

But we shall only consider the single-valued frames which (per definition) do not change on traversal of a closed path. These single-valued orthonormal basis systems we denote by

$$
\{\mid n, i: x>: i=1 \ldots \mathcal{N}\}
$$

They are transformed into each other by a $U(\mathcal{N})$ (or $O(\mathcal{N})-$ ) transformation $U_{i j}(x)$ which is a single-valued function of the parameter $x \in M$.

$$
\left|n, j ; x>=\sum_{i=1}^{\mathcal{N}}\right| n, i ; x>U_{i j}(x)
$$

These single-valued $U(\mathcal{N})$ (or $O(\mathcal{N})-$ ) transformations are the gauge transformations.[7]

The suitable mathematical framework for the description of $\mathcal{N}$-fold degenerate energy eigenstates is given by the $U(\mathcal{N})$ (or $O(\mathcal{N})$ ) principal fibre bundle over $M$, which in analogy to the B-S picture for the non-degenerate case will be denoted (collectively) by $\lambda_{\mathcal{N}}$ :

$$
\lambda_{\mathcal{N}}: U(\mathcal{N}) \rightarrow \ldots \rightarrow M
$$


The principal bundle corresponding to the A-A approach is now

$$
\eta_{\mathcal{N}}: U(\mathcal{N}) \rightarrow V_{\mathcal{N}, \mathcal{N}+k}(\mathbb{C}) \rightarrow G r_{\mathcal{N}, \mathcal{N}+k}(\mathbb{C})
$$

(or the corresponding real case with $U(\mathcal{N})$ replaced by $O(\mathcal{N})$ and $\mathbb{C}$ by $\mathbb{R}$ )

$$
\eta_{\mathcal{N}}: O(\mathcal{N}) \rightarrow V_{\mathcal{N}, \mathcal{N}+k}(\mathbb{R}) \rightarrow G r_{\mathcal{N}, \mathcal{N}+k}(\mathbb{R})
$$

where $G r_{\mathcal{N}, \mathcal{N}+k}(\mathbb{C})$ is the Grassmanian manifold of $\mathcal{N}$-dimensional subspaces or $\mathcal{N}$ dimensional projection operators in $\mathcal{H}=\mathbb{C}^{\mathcal{N}+k}\left(\mathcal{H}=\mathbb{R}^{\mathcal{N}+k}\right.$ for the real case $)$ and $V_{\mathcal{N}, \mathcal{N}+k}$ (C) is the manifold of $\mathcal{N}$-frames in $\mathbb{C}^{\mathcal{N}+k}$ (Stiefel manifold). 6 This is the $k$-universal bundle and $G_{\mathcal{N}, \mathcal{N}+k}$ is the corresponding classifying space for $U(\mathcal{N})$-resp. $O(\mathcal{N})$-principal bundles [15]. For an infinite-dimensional Hilbert space $\mathcal{H}$, one has to take the (inductive) limit $k \rightarrow \infty$,

$$
\eta_{\mathcal{N}}: U(\mathcal{N}) \rightarrow V_{\mathcal{N}}(C) \rightarrow G r_{\mathcal{N}}(\mathbb{C})
$$

We underline that there are subtleties in considering geometrical objects on the infinitedimensional spaces $V_{\mathcal{N}}(\mathbb{K})$ and $G r_{\mathcal{N}}(\mathbb{K}), \mathbb{K}=\mathbb{R}$ or $\mathbf{C}$. But, essentially, all the following considerations go through in the infinite-dimensional case.

$G r_{\mathcal{N}}(\mathbb{C})$ is the space of $\mathcal{N}$-dimensional (orthogonal) projection operators $\Lambda=\Lambda^{(\mathcal{N})}$, i.e. self-adjoint operators $\Lambda^{+}=\Lambda$ which fulfill $\Lambda^{2}=\Lambda \quad, \quad \operatorname{Tr} \Lambda=\mathcal{N} . V_{\mathcal{N}}(\mathbb{C})$ is the space of partial isometries, which are operators $v$ with the property that $v v^{+}=\Lambda$.[26-29] Physically $\Lambda^{(\mathcal{N})}$ represents quantum states that are not pure but mixed states (in which all the properties of $\Lambda \mathbb{H}$ appear with the same weight). They may, but need not be $\mathcal{N}$-fold degenerate energy eigenstates.

For the canonical projection $\pi_{\mathcal{N}}: V_{\mathcal{N}} \rightarrow G r_{\mathcal{N}}$ of the bundle (12) is given by

$$
\pi_{\mathcal{N}}(v)=v v^{+}=\Lambda^{(\mathcal{N})}
$$

In the special case $\mathcal{N}=1$ the bundle (10) is

$$
\eta_{1}=\eta: \quad U(1) \rightarrow S^{2 k+1} \rightarrow \mathbb{C} P^{k}=P(\mathcal{H}) ; \mathcal{H}=\mathbb{C}^{k+1}
$$

\footnotetext{
6 In distinction to the non-degenerate real case, the connection for the degenerate real case is not flat.
} 
where $\Lambda=\Lambda^{(1)}$ are the one-dimensional projections $\Lambda=|\chi><\chi|$ and $v$ is the map $v=\left|\chi><\chi^{(0)}\right|, \mid \chi^{(0)}>\in \mathcal{H}$ and $v^{+}=\left.\left|\chi^{(0)}><\chi\right|\right|^{7}$

The bundle of (10)-or of (12) for $k \rightarrow \infty$ - carries a natural connection, the Stiefel connection described by the connection form

$$
\tau=v^{+} d v
$$

If we choose a (local) section of $\eta_{\mathcal{N}}$ :

$$
G r_{\mathcal{N}} \supset \stackrel{S}{\mathcal{O}} V_{\mathcal{N}}
$$

given by the frame

$$
S(\Lambda)=\left\{\left|\phi^{1}(\Lambda)\right\rangle,\left|\phi^{2}(\Lambda)\right\rangle, \ldots,\left|\phi^{\mathcal{N}}(\Lambda)\right\rangle\right\}
$$

where $\mid \phi^{i}(\Lambda)>\in \mathcal{H}\left(=\mathbb{C}^{\mathcal{N}+k}\right)$ are orthonormal basis sections of $\eta_{\mathcal{N}}$ over $\Lambda \in G r_{\mathcal{N}}$ spanning $\mathcal{H}_{n}=\Lambda \mathcal{H}$ and fulfilling

$$
\left\langle\phi^{i}(\Lambda) \mid \phi^{j}(\Lambda)\right\rangle=\delta_{i j}
$$

then $\tau$ is (locally) represented by a $u(\mathcal{N})$-valued one-form $\mathcal{A}$ on $G r_{\mathcal{N}}$ :

$$
\mathcal{A}_{i j} \equiv\left(S^{*} \tau\right)_{i j}=\left\langle\phi^{i}|d| \phi^{j}\right\rangle
$$

Parallel transport with respect to $\tau$ in $\eta_{\mathcal{N}}$ has been discussed in several papers for the Abelian [4] and non-abelian [26-29] case. A closed curve

$$
\mathcal{C}:[0, T] \ni t \rightarrow \Lambda(t) \in O \subset G r_{\mathcal{N}} ; \Lambda(0)=\Lambda(T)
$$

is lifted into a curve for the initial frame $\left\{\mid \tilde{\psi}^{\mathbf{i}}(0)>\right\}=\left\{\mid \phi^{j}(\Lambda(0)>\}\right.$ :

$$
\tilde{\mathcal{C}}:[0, T] \ni t \rightarrow\left\{\left|\tilde{\psi}^{i}(t)\right\rangle: i=1,2 \ldots \mathcal{N}\right\} \quad ; \quad\left|\tilde{\psi}^{i}(T)\right\rangle=\sum_{j}\left|\tilde{\psi}^{j}(0)\right\rangle \mathcal{U}^{j i}
$$

7 In the finite dimensional case (10) when the $\mathcal{N}$-frame $v$ in $\mathbb{C}^{\mathcal{N}+k}$ is represented by $\mathcal{N} \times(\mathcal{N}+k)$-matrix and $v^{+}$by an $(\mathcal{N}+k) \times \mathcal{N}$-matrix, $v v^{+}$is represented by an $\mathcal{N} \times \mathcal{N}$ matrix and $v^{+} v$ by an $(\mathcal{N}+k) \times(\mathcal{N}+k)$ matrix. 
with the non-abelian phase factor given by

$$
\mathcal{U}^{i j}=\mathbb{P} \exp \left\{-\oint_{c} \mathcal{A}_{i j}\right\}
$$

The curve (20) is called the horizontal lift of the closed curve (19) and $\mathcal{U}^{j i}$ is called the holonomy or non-abelian Berry phase factor.

We come now to the relation between the bundle (12) in the A-A picture and the bundles (9) of the B-S picture.

As a generalization of the $\mathcal{N}=1$ case, (12) is the universal classifying bundle of $U(\mathcal{N})$ bundles over differentiable manifolds $M$. The $U(\mathcal{N})$ (or $O(\mathcal{N})$ ) bundles over $M$, (9), are classified by the homotopy set $\left[M, G r_{\mathcal{N}}\right]$. There is a one-to-one correspondence between the (set of isomorphism classes of) $U(\mathcal{N})$-bundles over $M$ and the homotopy classes of maps

$$
f: M \rightarrow G r_{\mathcal{N}}
$$

All bundles like (9) are obtained as pullbacks

$$
\lambda_{\mathcal{N}} \simeq f^{*}\left(\eta_{\mathcal{N}}\right)
$$

of $\eta_{\mathcal{N}}$ under $f$ : If $\hat{f}$ denotes the canonical $U(\mathcal{N})$ (or $O(\mathcal{N})$ ) bundle morphism induced by $f$, then

$$
\begin{array}{ccc}
f^{*}\left(\eta_{\mathcal{N}}\right) \cong \xi_{\mathcal{N}} & \stackrel{f}{\longrightarrow} & \eta_{\mathcal{N}} \\
\hat{S} \uparrow \mid \pi_{\xi} & & \downarrow \pi_{\eta} \uparrow \mathrm{S} \\
\mathbb{M} & \stackrel{f}{\longrightarrow} & G r_{\mathcal{N}}
\end{array}
$$

Each fibre of $\lambda_{\mathcal{N}}$ is homeomorphic to the corresponding fibre of $\eta_{\mathcal{N}}$ and sections of $\eta_{\mathcal{N}}$ pullback too: To the section $S$ of $(16)$ an associated section $\hat{S}: M \rightarrow f^{*}\left(\eta_{\mathcal{N}}\right)$ can be defined by the commutative diagram (24), $\hat{f} \circ \hat{S}=S \circ f$.

For the $U(\mathcal{N})$ bundle (9) of the B-S picture the map $f(x)$ is determined by the Hamiltonian $H(x)$ which associates to every $x \in M$ an eigenprojector $\Lambda(x) \in G r_{\mathcal{N}}$

$$
f(x)=\Lambda(x), x \in M \quad ; \quad H(x) \Lambda(x)=\Lambda(x) H(x)=E_{n}(x) \Lambda(x)
$$




$$
C:[0, T] \ni t \rightarrow x(t) \in M, x(T)=x(0)
$$

is a closed curve in parameter space $M$ with

$$
H(x(T))=H(x(0)) \quad \Lambda(x(T))=\Lambda(x(0))
$$

(because observables are single valued function of the parameters) then there is a corresponding closed curve (19) in the state space $G r_{\mathcal{N}}$ :

$$
\mathcal{C}:[0, T] \ni t \rightarrow \Lambda(t)=\Lambda(x(t))=(f \circ x)(t)
$$

The principal fibre bundle (9) of B-S is now given by

$$
\lambda_{\mathcal{N}}=f^{*}\left(r_{\mathfrak{N}}\right) \quad \text { with } \quad \mathfrak{f}^{*} \text { denoting the pullback }
$$

The section $\hat{S}=f^{*}(S \circ f)$ in this fibre bundle is given by the single valued basis system (8) (which is determined up to a gauge transformation $U_{i j}(x)$ ) :

$$
\hat{S}(x)=f^{*}(S(\Lambda(x)))=\{\mid n, i ; x>: i=1,2, \ldots \mathcal{N}\}
$$

which has the property

$$
\Lambda(x)=\sum_{i=1}^{\mathcal{N}}|n, i ; x\rangle\langle n, i ; x|
$$

with the association

$$
\left|\phi^{i}(\Lambda)\right\rangle \Longleftrightarrow|n, i ; x\rangle
$$

of (30) we obtain for the connection form $\hat{\tau}=f^{*} \tau$ on the bundle $\lambda_{\mathcal{N}}=f^{*}\left(\eta_{\mathcal{N}}\right)$

$$
A_{i j}(x) \equiv\left(\hat{S}^{*} \hat{\tau}\right)_{i j}=\left\langle n, i ; x|d| r_{i} j ; x\right\rangle=f^{*} \mathcal{A}_{i j}
$$

This relates the (non-abelian) A-A connection $\mathcal{A}_{i j}$ to the (non-abelian) Berry connection $A_{i j}$.

A particularly interesting case is presented in [12] for the fermionic time reversal invariant systems. Here $T=-1$; and $i, T$, and $i T$ endow the Hilbert space with a quaternionic structure (see also DYSON [30]). 8 The A-A bundle for this case:

$$
S p(1) \rightarrow S^{4 k+3} \rightarrow \mathbb{H} P(k) \quad, \quad k \rightarrow \infty
$$

8 The unavoidable two-fold complex degeneracy is called the "Kramer's degeneracy". 
has $S p(1) \cong S U(2)$ as its structure group. Since in general,

$$
\pi_{i}(\mathbb{H} P(\infty)) \cong \pi_{i-1}(S U(2))
$$

the first non-trivial homotopy group of $\mathbb{H} P(\infty)$ is:

$$
\pi_{4}(\mathbb{H} P(\infty)) \cong \pi_{3}(S U(2))=\pi_{3}\left(S^{3}\right) \cong \mathbb{Z}
$$

However as $S^{3}$ has higher homotopy groups $\mathbb{H} P(\infty)$ is not an Eilenberg-McLane space. The maps $f: m \rightarrow \mathbb{H} P(\infty)$ induce $S p(1)$-bundles over $M$. The bundles have well-defined second Chern classes $\left(\in H^{4}(m, \mathbb{Z})\right)$, which however do not classify all the $S p(1)$-bundles.

\section{THE NON-ADIABATIC CASE}

In the A-A picture the projection operators $\Lambda \in G r_{\mathcal{N}}$ represent $\mathcal{N}$-fold degenerate physical (not necessarily energy eigen-) states (of a given Hamiltonian for a particular physical system).

If a Hamiltonian $H(t)$ is given then the time evolution of a state $W(t)$ is described by (a generalization of the basic postulate for time evolution [31] section XII.1) the time dependent Schrödinger (also called von Neumann) equation

$$
i \frac{d}{d t} W(t)=[H(t), W(t)],
$$

where $W(t)$ is in general a statistical operator. For $\mathcal{N}=1, W(t)=|\psi(t)><\psi(t)|$ where $\mid \psi(t)>$ fulfills the ordinary time dependent Schrödinger equation. We will restrict ourselves to $W(0)=\Lambda^{\mathcal{N}}(0)$, where $\Lambda^{\mathcal{N}}$ are $\mathcal{N}$-dimensional orthogonal projection operators. Then any curve (19) closed $(\Lambda(0)=\Lambda(T))$ or non-closed $(\Lambda(0) \neq \Lambda(T))$ can be a curve of time-development of a physical state

$$
t \rightarrow W(t) \quad, \quad W(0)=\Lambda^{\mathcal{N}}(0)
$$

i.e. be a solution of (33).

For a given Hamiltonian $H(t)$ not every $\Lambda^{\mathcal{N}}(0)$ will be the initial state, $W(0)=\Lambda^{\mathcal{N}}(0)$, of a solution of (33) which is a closed curve (19):

$$
\Lambda^{\mathcal{N}}(0)=W(0)=W(T) \equiv \Lambda^{\mathcal{N}}(T)
$$


But there are (probably a discrete number of) initial states $W^{c y c l i c}(0)=\Lambda^{\mathcal{N}}(0)$ which are cyclic i.e. there are $W^{\text {cyclic }}(t)$ which develop according to (33) along closed curves (19) [32]. The majority of solutions of (33) however are non-cyclic.

In the B-S picture the Hamiltonian $H(x(t))$ depends smoothly on the parameter $x \in M$ and associates to every $x \in M$ an eigenstate $\Lambda_{n}(x) \in G r_{\mathcal{N}}$

$$
\forall x \in M \quad H(x) \Lambda_{n}(x)=\Lambda_{n}(x) H(x)=E_{n}(x) \Lambda_{n}(x)
$$

To a close curve in parameter space

$$
C:[0, T] \ni t \rightarrow x(t) \in M \quad, \quad x(T)=x(0)
$$

corresponds a closed curve (19) in $G r_{\mathcal{N}}$

$$
\mathcal{C}:[0, T] \ni t \rightarrow \Lambda_{n}(t)=\Lambda_{n}(x(t)) \quad, \quad \Lambda_{n}(x(0))=\Lambda_{n}(x(T))
$$

(Because observables like $H$ and $\Lambda_{n}$ are single valued functic..s of the parameters $H(x(0))=H(x(T))$. The adiabatic evolution of a state $W(0)=\Lambda_{n}(x(0))$ is defined to be such that at all times (due to the gentle change of $H(t)$ ):

$$
W(t)=\Lambda_{n}(x(t))
$$

An adiabatic evolution (39) would therefore (because of (38)) be a cyclic evolution.

It is easy to see that an adiabatic evolution does not exist and (39) can only be an approximation. Because, if (39) would fulfill (33), then we would obtain as a consequence of $(36)$ :

$$
i \frac{d}{d t} W(t)=\left[H(x(t)), \Lambda_{n}(x(t))\right]=0
$$

Thus any adiabatic evolution would be a stationary evolution $W(t)=W(0)$. In other words the energy eigenstate $\Lambda_{n}(x(0))$ can not be the initial state of a cyclic evolution $W^{\text {cyclic }}(0)$ and for a $W(t)$ fulfilling (33) one has only

$$
W(0) \approx \Lambda_{n}(x(0))=\Lambda_{n}(x(T)) \approx W(T)
$$


As the eigenstates $\Lambda_{n}(x)$ of the Hamiltonian $H(x)$ do not provide cyclic states, we look for a diffeomorphism

$$
F: M \rightarrow M
$$

such that

$$
\tilde{H}(x) \equiv H(F(x))
$$

has an $\mathcal{N}$-dimensional eigenprojector $\tilde{\Lambda}_{n}$, which is a cyclic solution of (33). We will restrict ourselves here to diffeomorphisms $F$ which are homotopic to the identity. Whether such a diffeomorphism $F$ exists depends upon the physical parameters of the Hamiltonian (like its angular frequency $\omega=\frac{2 \pi}{T}$ and some internal frequency):

$$
\begin{aligned}
& {[0, T] \ni t \rightarrow W(t)=\tilde{\Lambda}_{n}(x(t))} \\
& W(0)=\tilde{\Lambda}_{n}(x(0))=\tilde{\Lambda}_{n}(x(T))=W(T)
\end{aligned}
$$

Then the map

$$
\tilde{f}: M \ni x \rightarrow \tilde{\Lambda}_{n}(x) \in G r_{\mathcal{N}}
$$

which associates to every $x \in M$ an eigenstate $\tilde{\Lambda}_{n}(x) \in G r_{\mathcal{N}}$ of $\tilde{H}(x)$ and which associates to a closed curve in $M(37)$, a closed curve of states $W(t)=\tilde{\Lambda}_{n}(x(t))$ (fulfilling (33)) in $G r_{\mathcal{N}}$, can be used to construct a bundle over the parameter space.

The $U(\mathcal{N})(O(\mathcal{N}))$ bundle over $M$ is now given by

$$
\tilde{\lambda}_{\mathcal{N}}=\tilde{f}^{*}\left(\eta_{\mathcal{N}}\right)
$$

with $\tilde{f}^{*}$ being the pull-back with respect to $\tilde{f}$.

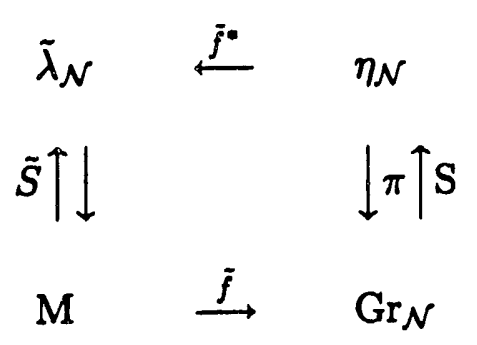

The (local) section $\tilde{S}=\tilde{f}^{*}(S \circ \tilde{f})$ in this fibre bundle $\tilde{f}^{*}\left(\eta_{\mathcal{N}}\right)$ is given by the single valued basis system associated to

$$
\begin{aligned}
\tilde{S}(x) & =\tilde{f}^{*}(S(\tilde{\Lambda}(x)))=\left\{\tilde{f}^{*}\left(\mid \phi^{1}(\tilde{\Lambda})>\right), \ldots, \tilde{f}^{*}\left(\mid \phi^{\mathcal{N}}(\tilde{\Lambda})>\right)\right\} \\
& \equiv:\{|\tilde{\phi}, 1 ; x>; \ldots,| \tilde{\phi}, \mathcal{N} ; x>\}
\end{aligned}
$$


It consists of eigenvectors $\mid \tilde{\phi}, i ; x>$ of $\tilde{H}(x) \cdot \mid \tilde{\phi}, i ; x>$ are determined up to a gauge transformation $U_{i j}(x)$ and have the following property:

$$
\tilde{\Lambda}_{n}(x)=\Sigma_{i}\left|\tilde{\phi}, i ; x><\tilde{\phi}_{,} ; x\right|
$$

The canonical connection one-form $\tilde{\tau}=\tilde{f}^{*} \tau$ on the bundle $\tilde{\lambda}_{\mathcal{N}}=\tilde{f}^{*}\left(\eta_{\mathcal{N}}\right)$, is obtained from the canonical connection on the Stiefel bundle (14), which is locally represented by the one-form (18). It is therefore given (locally) by

$$
\tilde{A}_{i j}(x) \equiv\left(\tilde{S}^{*} \tau\right)_{i j}=<\tilde{\phi}, i ; x\left|\frac{\partial}{\partial x^{\mu}}\right| \tilde{\phi}, j ; x>d x^{\mu}=\tilde{f}^{*} \mathcal{A}_{i j}
$$

The non-abelian geometric phase factor (21) can then be rewritten as follows

$$
\mathcal{U}^{i j}=\mathbb{P} \exp \left\{-\oint_{C=\tilde{f} \circ C} \mathcal{A}_{i j}\right\}=\mathbb{P} \exp \left\{-\oint_{C} \tilde{A}_{i j}(x)\right\}
$$

where $C$ is the close curve (37) in $M$ and $\mathcal{C}$ the corresponding (by (45)) curve in $G r_{\mathcal{N}}$.

This means that $\mathcal{U}$ can be interpreted as the (non-abelian) phase factor acquired after parallel transport with respect to $\tilde{f}^{*} \mathcal{A}$ along the closed curve $C$ in parameter space $M$. Formula (51) says that the geometric phase can be either calculated using the canonical connection $\tau$ in the universal bundle $\eta_{\mathcal{N}}$ or using its pull-back $\tilde{f}^{*} \tau$ on the induced bundle $\tilde{\lambda}_{\mathcal{N}}$

The (non-abelian) Berry connection and phase of Wilczek and Zee [33] is obtained from (50) in the (adiabatic) approximation in which $F$ of (43) is replaced by the identity map. To discuss this in more detail we remark that (43) implies

$$
\tilde{\Lambda}_{n}(x)=\Lambda_{n}(F(x)) \text { and } \quad|\tilde{\phi}, i ; x>=| n, i ; F(x)>
$$

where $\Lambda_{n}(x)$ are the eigenprojectors (36) of $H(x)$ corresponding to the eigenvalues $E_{n}(x)$ and $\{\mid n, i ; x>\}$ is the single valued basis system (7) spanning $\Lambda_{n}(x) \mathcal{H}=\mathcal{H}_{n}(x)$. The map

$$
f=\tilde{f} \circ F^{-1}: M \ni x \rightarrow \Lambda_{n}(x) \in G r_{\mathcal{N}}
$$

induces then an isomorphism between the A-A bundle (10) (or(12)) and B-S bundle (9) as discussed in Section IV and illustrated in the following diagram:

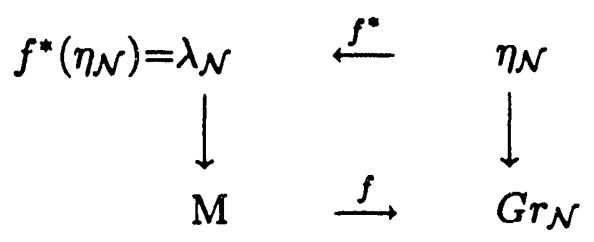


As $F$ is a diffeomorphism ${ }^{9}$ (per assumption) the bundle morphism $F^{*}: \lambda \rightarrow \tilde{\lambda}=F^{*} \lambda$ induced by $F$ is an isomorphism. This means that $\lambda$ and $\tilde{\lambda}$ have the same topology since $f$ and $\tilde{f}$ belong to the same homotopy class in $\left[M, G r_{\mathcal{N}}\right]$.

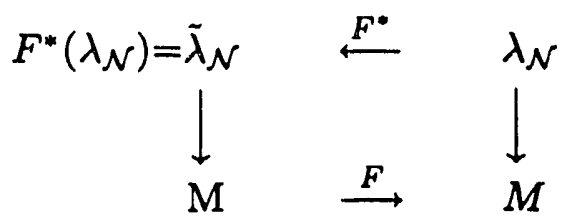

If $F$ fails to be a diffeomorphism for some values of the physical parameters of the Hamiltonian $H$ (but still fulfil (43), (53)), then the topology of $\tilde{\lambda}$ and $\lambda$ can differ. The detailed analysis for a particular example of this will be discussed in a separate paper [31].

Parallel transport can be defined in two alternative ways. There is first the connection (50) given by the canonical connection on the Stiefel bundle (14). Using (52), the geometric phase factor (51) is given by:

$$
\mathcal{U}^{i j}=\mathbb{P} \exp \left\{-\oint_{C}<n, i ; F(x)\left|\frac{\partial}{\partial x^{\mu}}\right| n, j ; F(x)>d x^{\mu}\right\}
$$

In addition to (50) one can define the (non-abelian) Berry connection (locally) by:

$$
A_{i j}(x) \equiv<n, i ; x\left|\frac{\partial}{\partial x^{\mu}}\right| n, j ; x>d x^{\mu}
$$

The (non-abelian) Berry phase acquired after parallel transport with respect to the Berry connection (55) is

$$
\mathcal{U}_{\text {Berry }}^{i j}=\mathbb{P} \exp \left\{-\oint_{C}<n, i ; x\left|\frac{\partial}{\partial x^{\mu}}\right| n, j ; x>d x^{\mu}\right\}
$$

In (54) and (56), $C$ is the close curve (37) in $M$. Only in the case that the map $F$ becomes the identity do the Berry connection (55) and Aharonov-Anandan connection (50) agree.

Our arguments in here are based on the existence of the map $F$ for a given cyclic Hamiltonian $H(x(t))$. The necessary and sufficient conditions for the existence of $F$ for general cyclic Hamiltonians are not known, but there are examples where $F$ exists and our analysis applies [31].

${ }^{9} F$ is a diffeomorphism which is homotopic to the identity map. 


\section{Conclusion}

The universal classifiying $U(\mathcal{N})$ bundle (12) carries a natural connection, the Stiefel connection. For $\mathcal{N}=1$ this Stiefel connection of mathematics is identical with the Aharonov-Anandan connection which was originally obtained by separating from the total phase factor for non-adiabatic cyclic evolution of a statevector $\mid \psi(t)>$ the "dynamical" part $\exp \left[-\frac{i}{\hbar} \int_{0}^{T}\langle\psi(t)|H(t)| \psi(t)\rangle d t\right]$ and interpreting the reminder as the "geometrical" part $\exp \left[i \int_{0}^{T} A\right]$ defined by the $A-A$ connection $A$. This brings the physics of cyclic quantum evolution into correspondence with the mathematics of the classification theorem for vector bundles. These considerations provide a straightforward topological classification of the geometric quantum phase. Furthermore, they suggest a procedure for constructing a parameter space bundle for exact cyclic evolution of a Hamiltonian which is a function over this parameter space.

The Stiefel connection (or Aharonov-Anandan connection in the abelian case) goes into the (abelian) Berry connection when the exact cyclic evolution becomes adiabatic. Vice versa, the (non-abelian) Berry connection of adiabatic evolution can be related to an exact cyclic evolution. An example, in which the relation between the Aharonov-Anandan and the Berry connection is discussed in all details will be presented in a following paper [34]. 


\section{References:}

[1.] B. Simon: Phys. Rev. Lett. 51, 2167 (1983).

[2.] M. Berry: Proc. R. Soc. Lond. A392, 45 (1984).

[3.] Y. Aharonov and J. Anandan: Phys. Rev. Lett. 58, 1593 (1987).

[4.] J. Anandan and Y. Aharonov: Phys. Rev. D38, 1893 (1988); J. Anandan: Ann. Inst. Henri Poincaré 49, 271 (1988).

[5.] J. Samuel and Bhandari, R.: Phys. Rev. Lett. 60, 2339 (1988).

[6.] A. Bohm, L.J. Boya and B. Kendrick: Phys. Rev. A43, (1991).

[7.] A. Bohm, L.J. Boya, B. Kendrick and M. Loewe: J. Math. Phys.

[8.] A. Bohm, L.J. Boya, and B. Kendrick: "On the Uniqueness of the Geometric (Berry) Connection," University of Texas preprint, June (1991).

[9.] L.J. Boya, J. Carinena, and Gracia-Bondia, J.M.: "Symplectic Approach to the Aharonov-Anandan (Geometric) Phase." U. Zaragoza (Spain) preprint, July (1991).

[10.] E. Kiritsis, : Commun. Math. Phys. 111, 417 (1987).

[11.] R.R. Douglas, A.R. Rutherford (1990) "Quantum Adiabatic Phase, Algebraic Topology, and Time-Reversal Invariance." Lecture Notes in Physics. New York: SpringerVerlag (in press).

[12.] J.E. Avron, L. Sadun, J. Segret, and B. Simon: Phys. Rev. Lett. 61, 1329 (1988) Commun. Math. Phys. 124, 595 (1989).

[13.] J. Anandan and L. Stodosky, Phys. Rev. D35, 2597 (1987).

[14.] J. Milnor, and J.D. Stasheff, "Characteristic Classes," Princeton University Press, Princeton, NJ (1974).

[15.] N. Steenrod, "The Topology of Fibre Bundles." Princeton University Press, Princeton, NJ (1951).

[16.] H. Whitney, Bull. Ann. Math. Soc. 43, 785 (1937).

[17.] B. Kostant, "Quantization and Unitary Representations," in "Lectures in Modern Analysis and Applications," Lecture Notes in Math 170, Springer-Verlag (1970), p. 87.

[18.] R. Bott, and L.W. Wu, "Differential Forms in Algebraic Topology," Springer-Verlag, Berlin (1982). 
[19.] S. Eilenberg, N. Steenrod, "Foundations of Algebraic Topology," Princeton Math. Ser. 15, Princeton University Press (1952).

[20.] F. Wilczek, and A. Shapere, Geometric Phases in Physics, World Scientific, Singapore (1990), Chapter 2.

[21.] L.J. Boya and J. Mateos, J. Phys. A (1980).

[22.] L.H. Ryder, Eur. J. Phys. (GB) 3, no. 4, p. 206-9 (1982).

[23.] M. Narasimhan and S. Ramanan, Amer. J. Math. 83, 563 (1961).

[24.] G. Herzberg, and H.C. Longuet-Higgins, Disc. Farad. Soc. 35, 77 (1963).

[25.] S. Kobayashi, and K. Nomizu, "Foundations of Differential Geometry," WileyInterscience, New York, Vol. 1 (1963), Vol. 2 (1969).

[26.] A. Uhlmann, Rep. Math. Phys. 24, 229 (1986) and "Parallel Transport of Phases," in "Differential Geometry, Group Representation and Quantization," J.D. Henning, W. Lucke, J. Tolar, Lecture Notes in Physics 379, Springer-Verlag (1991).

[27.] J. Dittmann, and G. Rudolph, "On a Connection Governing Parallel Transport Along $2 \times 2$-Density Matrices," Uni. Leipzig preprint (1992); subrnitted to J. Geom. Phys.

[28.] L. Dabrowski, H. Grosse, "Quantum Holonomy for Mixed States," University vienna preprint UWThPh-1988-36.

[29.] L. Dabrowski, ICTP Trieste preprint 156/88.

[30.] F.J. Dyson, J. Math. Phys. 3, 140 (1964).

[31.] A. Bohm, "Quantum Mechanics: Foundations and Applications," Third Edition, Springer-Verlag, to appear; A. Bohm, "Quantum Phase Factors and Their Conseqences," (in preparation).

[32.] D.J. Moore, Phys. Rep. 210, No. 1 (1991) 1-43.

[33.] F. Wilczek, and A. Zee, Phys. Rev. Lett. 52, 2111 (1984).

[34.] A. Mostafazadeh, and A. Bohm, "Topology of the Parameter Space Bundle and NonAdiabatic Berry Phase," (in preparation). 

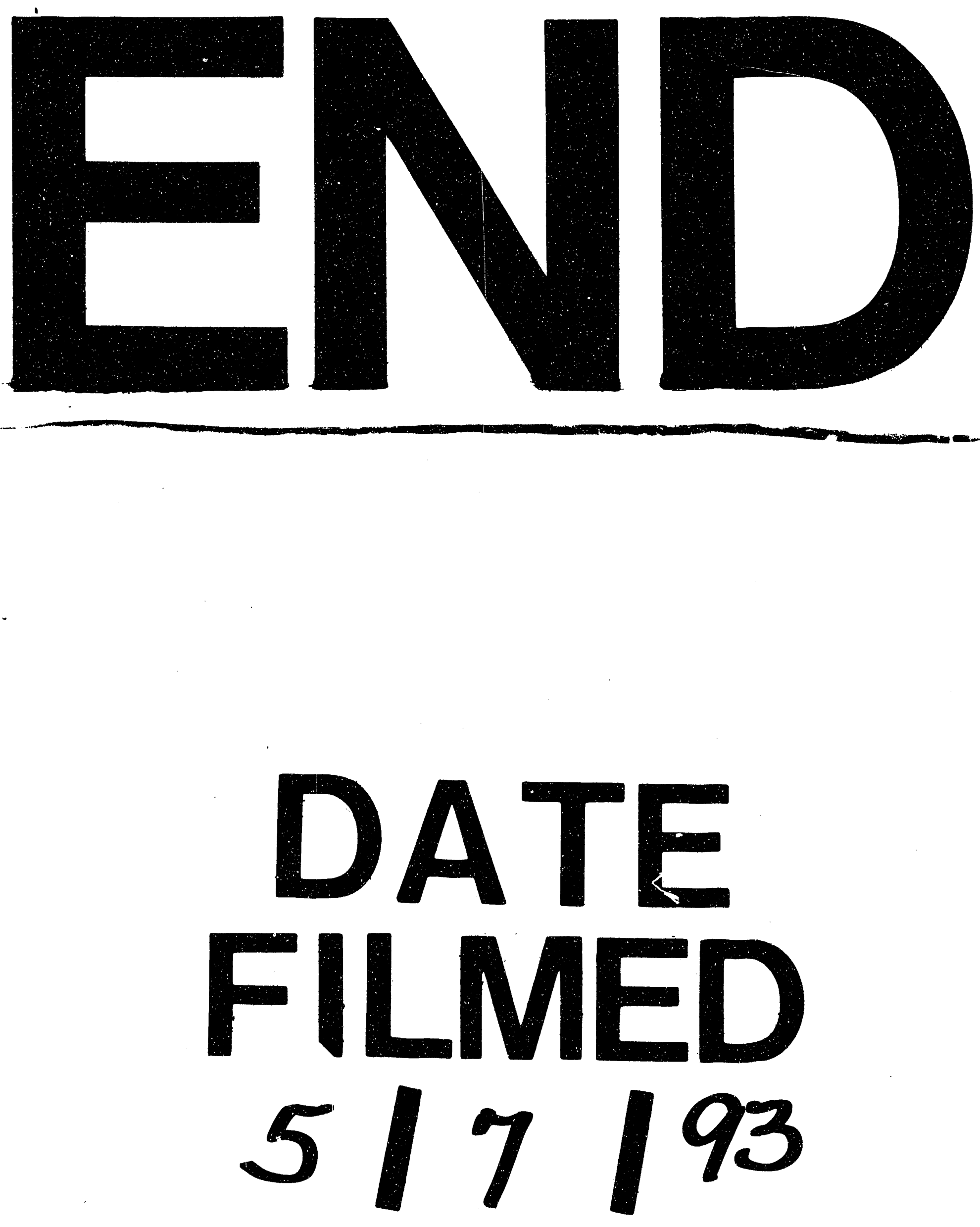
